\title{
Association of doublecortin-like kinase I with tumor aggressiveness and poor biochemical recurrence-free survival in prostate cancer
}

This article was published in the following Dove Press journal: OncoTargets and Therapy

\author{
Donggen Jiangl,* \\ Chutian Xiaol,* \\ Tuzeng Xian' \\ Liantao Wang ${ }^{2}$ \\ Yunhua Mao' \\ Junfu Zhang' \\ Jun Pang ${ }^{1,3}$
}

'Department of Urology, the Third Affiliated Hospital of Sun Yat-Sen University, Guangzhou, China; ${ }^{2}$ Department of General Surgery, Shenzhen Shajing Affiliated Hospital of Guangzhou Medical University, Shenzhen, China; ${ }^{3}$ Department of Urology, the Seventh Affiliated Hospital of Sun Yat-Sen University, Shenzhen, China

*These authors contributed equally to this work

\begin{abstract}
Background: Doublecortin-like kinase 1 (DCLK1) has been proven to be involved in numerous tumors, while its role in prostate cancer (PCa) is still unclear. This study aimed at investigating the expression pattern and prognostic value of DCLK1 in PCa.

Patients and methods: Real-time polymerase chain reaction and Western blot were employed to determine DCLK1 mRNA and protein levels in 25 paired fresh samples of $\mathrm{PCa}$ and benign prostatic hyperplasia (BPH) as well as in PCa cell lines. Immunohistochemistry (IHC) was also performed in $125 \mathrm{PCa}$ and $65 \mathrm{BPH}$ tissues to assess DCLK1 expression. Then, the association of DCLK1 expression with clinicopathological parameters and biochemical recurrence (BCR) after radical prostatectomy was statistically analyzed. In addition, the role of DCLK1 in PCa cell proliferation, migration, and invasion was evaluated by using MTT and transwell assays.

Results: The mRNA and protein levels of DCLK1 were markedly higher in the fresh samples of PCa than that in BPH. Consistently, IHC revealed increased expression of DCLK1 in PCa paraffin-embedded tissues compared with BPH. Moreover, increased DCLK1 expression was significantly associated with postoperative Gleason grading $(P=0.012)$, pathological $\mathrm{T}$ stage $(P=0.001)$, seminal vesicle invasion $(P=0.026)$, and lymph node involvement $(P=0.017)$, respectively. The Kaplan-Meier curve analysis demonstrated that high DCLK1 expression was associated with lower postoperative BCR-free survival (bRFS). Furthermore, multivariate Cox analysis showed that postoperative Gleason grading $(P=0.018)$, pathological T stage $(P<0.001)$, seminal vesicle invasion $(P=0.012)$, lymph node involvement $(P=0.014)$, and DCLK1 expression $(P=0.014)$ were independent predictors of BCR. In vitro, the overexpression and knockdown of DCLK1 in PCa cell lines indicated that DCLK1 could promote cell proliferation, migration, and invasion.
\end{abstract}

Conclusion: Increased DCLK1 expression is associated with PCa aggressiveness and may independently predict poor bRFS in patients with $\mathrm{PCa}$.

Keywords: prostatic neoplasm, DCLK1, BCR, prognosis, radical prostatectomy, biomarker

\section{Introduction}

The incidence of prostate cancer ( $\mathrm{PCa}$ ) in China is increasing steadily in recent years, with estimated 60,300 new cases and 26,600 deaths of PCa in 2015. ${ }^{1}$ As a clinically heterogeneous multifocal disease, up to $40 \%$ of PCa treated by radical prostatectomy (RP) may undergo biochemical recurrence (BCR) at a long-term follow-up. ${ }^{2}$ Although diagnostic tools, including prostate-specific antigen (PSA), pathological $\mathrm{T}$ stage, and Gleason score, were traditionally recognized as prognostic factors for $\mathrm{PCa}$, their accuracies may have limitations. ${ }^{3}$ Therefore, novel effective prognostic biomarkers for
Correspondence: Jun Pang Department of Urology, the Third Affiliated Hospital of Sun Yat-Sen University, No 600 Tianhe Road,

Guangzhou 510630, China

Tel +862085252990

Fax +86 2085252678

Email pangjun530@I63.com
OncoTargets and Therapy 2018:1 I 1077-1086 Dovepress in http://dx.doi.org/10.21 147/OTT.SI57295 (c) (1) (5) 2018 jiang et al. This work is published and licensed by Dove Medical Press limited. The full terms of this license are available at https://www.dovepress.com/terms.php (c)
hereby accept the Terms. Non-commercial uses of the work are permitted without any further permission from Dove Medical Press Limited, provided the work is properly attributed. For permission for commercial use of this work, please see paragraphs 4.2 and 5 of our Terms (https://www.dovepress.com/terms.php). 
BCR after RP are required to provide valuable information on PCa risk, recurrence, and prognosis clinically.

Doublecortin-like kinase 1 (DCLK1) is a serine/threonine protein kinase that belongs to the family of microtubuleassociated proteins. DCLK1 was initially reported to play a pivotal role in neuronal migration and neurogenesis. ${ }^{4}$ Accumulating evidences have demonstrated the overexpression of DCLK1 in numerous human cancers, including colon, ${ }^{5,6}$ pancreatic, ${ }^{6}$ liver, ${ }^{7}$ kidney, ${ }^{8}$ and esophageal cancers. ${ }^{9}$ DCLK1 has also been proposed as a marker of intestinal and pancreatic cancer stem cells (CSCs), and its overexpression is critical for cancer initiation, growth, stemness, epithelial-mesenchymal transition (EMT), and metastasis. ${ }^{5-8}$ However, to the best of our knowledge, little is known about the association between DCLK1 and PCa; the expression and prognostic implications of DCLK1 in PCa have not yet been fully elucidated.

In the present study, quantitative real-time polymerase chain reaction (qRT-PCR), Western blot, and immunohistochemistry (IHC) were performed to assess DCLK1 expression in tissue samples and PCa cell lines. We then analyzed the association between DCLK1 expression and clinicopathological features of the patients who underwent RP. We, for the first time, investigated the role of DCLK1 in PCa cell proliferation, migration, and invasion. Overall, our study revealed that DCLK1 expression was significantly upregulated in $\mathrm{PCa}$ compared with benign prostatic hyperplasia (BPH). The increased DCLK1 expression was associated with PCa tumor aggressiveness and might efficiently predict poor BCR-free survival (bRFS) in patients after RP.

\section{Patients and methods}

\section{Patients and tissue samples}

A total of 125 archived paraffin-embedded tissues from $\mathrm{PCa}$ patients after RP and $65 \mathrm{BPH}$ tissues from transurethral resection of the prostate were obtained from the Department of Pathology, the Third Affiliated Hospital of Sun Yat-Sen University, between January 2008 and June 2010. None of the PCa patients had received chemotherapy, radiotherapy, or hormonal therapy prior to the RP. All the tissues were processed in a comparable manner and histologically reviewed by two pathologists. The tumors were staged by the American Joint Committee on Cancer 8th Edition Prostate Cancer Staging Classification ${ }^{10}$ and graded according to the 2014 International Society of Urological Pathology guidelines. ${ }^{11}$ The patients were followed up from the date of surgery to the date of death or the last follow-up, and BCR was defined as two consecutive serum PSA levels $>0.2 \mathrm{ng} / \mathrm{mL}$ after RP. ${ }^{12}$
Table I Correlation between DCLKI expression and clinicopathological parameters of the 125 patients with $\mathrm{PCa}$

\begin{tabular}{|c|c|c|c|c|}
\hline \multirow[t]{2}{*}{ Variables } & \multirow[t]{2}{*}{ Total (\%) } & \multicolumn{2}{|c|}{ DCLKI expression } & \multirow[t]{2}{*}{$P$-value } \\
\hline & & Low (\%) & High (\%) & \\
\hline All cases & $125(100)$ & $53(100)$ & $72(100)$ & - \\
\hline Age (years) & & & & 0.799 \\
\hline$<68$ & $62(48.8)$ & $27(50.9)$ & $35(48.6)$ & \\
\hline$\geq 68$ & $63(5 \mid .2)$ & $26(49.1)$ & $37(51.4)$ & \\
\hline \multicolumn{2}{|l|}{ Preoperative PSA (ng/mL) } & & & 0.066 \\
\hline$<10$ & $21(16.8)$ & $14(26.4)$ & $7(9.7)$ & \\
\hline $10-20$ & $47(37.6)$ & $18(34.0)$ & $29(40.3)$ & \\
\hline$>20$ & $57(45.6)$ & $21(39.6)$ & $36(50.0)$ & \\
\hline \multicolumn{2}{|c|}{ Postoperative Gleason grading } & & & 0.012 \\
\hline Grade group I & $53(42.4)$ & $30(56.6)$ & $23(32.0)$ & \\
\hline Grade groups 2 and 3 & $38(30.4)$ & $12(22.6)$ & $26(36.0)$ & \\
\hline Grade groups 4 and 5 & $34(27.2)$ & II (20.8) & $23(32.0)$ & \\
\hline Pathological T stage & & & & 0.001 \\
\hline pT2 & $75(60.0)$ & $40(75.5)$ & $35(48.6)$ & \\
\hline PT3a & $33(26.4)$ & $10(18.9)$ & $23(32.0)$ & \\
\hline pT3b & $17(13.6)$ & $3(5.6)$ & $14(19.4)$ & \\
\hline Seminal vesicle invasion & & & & 0.026 \\
\hline Yes & $17(13.6)$ & $3(5.66)$ & $14(19.44)$ & \\
\hline No & $108(86.4)$ & $50(94.34)$ & $58(80.56)$ & \\
\hline Lymph node involvement & & & & 0.017 \\
\hline Yes & $24(19.2)$ & $5(9.4)$ & $19(26.4)$ & \\
\hline No & I0I (80.8) & $48(90.6)$ & $53(73.6)$ & \\
\hline Positive surgical margin & & & & 0.909 \\
\hline Yes & $23(18.4)$ & $10(18.9)$ & $13(18.1)$ & \\
\hline No & $102(81.6)$ & 43 (8I.I) & 59 (81.9) & \\
\hline
\end{tabular}

Abbreviations: DCLKI, doublecortin-like kinase I; PCa, prostate cancer; PSA, prostate-specific antigen.

Demographic and clinicopathological data of the patients were obtained from their medical records and reassessed (Table 1). Besides, for qRT-PCR and Western blot analysis, 25 paired fresh $\mathrm{PCa}$ and $\mathrm{BPH}$ specimens were collected randomly and stored in liquid nitrogen immediately after the surgery between January 2016 and December 2016. This study was approved by the Ethics Committee of the Third Affiliated Hospital of Sun Yat-Sen University (Number: [2015] 2-130), with a Research Registry Unique Identifying Number (researchregistry2902). Written consent from the study population was considered, but as this was a retrospective study where most of the materials were $>5$ years old and all data were analyzed anonymously, it was considered not needed.

\section{$\mathrm{IHC}$ and scoring}

Formaldehyde-fixed and paraffin-embedded specimens were cut into $4-\mu \mathrm{m}$ serial sections. Briefly, tissue sections were deparaffinized in xylene, rehydrated with ethanol, and then incubated in 3\% hydrogen peroxide for 10 minutes to halt endogenous peroxidase activity. After being heated in 
$0.01 \mathrm{M}$ citrate buffer $(\mathrm{pH}=6.0)$ for 15 minutes at $100^{\circ} \mathrm{C}$ in a microwave oven to repair the tissue antigen, the sections were incubated overnight at $4{ }^{\circ} \mathrm{C}$ with polyclonal rabbit antiDCLK1 antibody (1:100, AP7219b; Abgent, San Diego, CA, USA), followed by secondary antibody incubation for 30 minutes at $37^{\circ} \mathrm{C}$. The sections were semi-quantitatively scored according to a previous report ${ }^{13}$ as follows: staining intensity was scored as 0 (no staining), 1 (faint/equivocal or focal staining), 2 (intermediate staining), and 3 (bright staining), multiplied by the percentage of cells at each immunostaining level, resulting in a total score range of 0-300. The total DCLK1 expression score of each section was defined as follows: 0, negative; 1-100, weak; 101-200, moderate; and 201-300, strong. For further analysis, the IHC result of each patient was finally considered as low DCLK1 expression (total expression score, 0-100) or high DCLK1 expression (total expression score, 101-300).

\section{Cell lines and cell culture}

Human PCa cell lines (LNCaP, PC3, DU145, and 22Rv1) and a prostatic normal epithelial cell line (RWPE-1) were obtained from the Institute of Biochemistry and Cell Biology of the Chinese Academy of Sciences (Shanghai, China). The PCa cells were cultured in Dulbecco's Modified Eagle's Medium (Thermo Fisher Scientific, Waltham, MA, USA) supplemented with $10 \%$ fetal bovine serum (FBS), $100 \mu \mathrm{g} / \mathrm{mL}$ streptomycin, and $100 \mathrm{U} / \mathrm{mL}$ penicillin in a humidified incubator at $37^{\circ} \mathrm{C}$ containing $5 \% \mathrm{CO}_{2}$, while RWPE- 1 cells were cultured in a defined keratinocyte serum-free medium with growth supplement (Thermo Fisher Scientific) in the same condition mentioned above.

\section{RNA extraction and qRT-PCR}

Total RNA from tissues and cells was extracted by using TRIzol reagent (Invitrogen, Grand Island, NY, USA) following the manufacturer's instructions. The cDNA was synthesized from RNA samples with the Transcriptor First-Strand cDNA Synthesis Kit (Roche, Mannheim, Germany). qRT-PCR analysis was performed on LightCycler ${ }^{\circledR} 480$ System (Roche) using an SYBR Green I Master Kit (Roche). A human glyceraldehyde-3-phosphate dehydrogenase (GAPDH) gene was used as an endogenous control for sample normalization. The primers used were as follows: forward: $5^{\prime}$-CTGGGCT ACACTGAGCACC-3' and reverse: 5'-AAGTGGTCGTT GAGGGCAATG-3' for human GAPDH; and forward: 5'-CAGCAACCAGGAATGTATTGGA-3' and reverse: 5'-CTCAACTCGGAATCGGAAGACT-3' for human
DCKL1. The relative DCKL1 mRNA levels were analyzed by calculating the $2^{-\Delta \Delta \mathrm{Ct}}$ values.

\section{Western blot}

Total proteins were extracted by using radioimmunoprecipitation assay (Cell Signaling Technology, Danvers, MA, USA) containing protease inhibitors. Protein levels were measured by Bradford method with the BCA Protein Assay Kit (Thermo Fisher Scientific). Equal amounts of $40 \mu \mathrm{g}$ respective tissue proteins were separated by $12 \%$ sodium dodecyl sulfatepolyacrylamide gel electrophoresis and then transferred to polyvinylidene fluoride membranes (Millipore Corporation, Billerica, MA, USA). After blocked by 5\% nonfat milk for 1 hour, the membranes were incubated overnight at $4{ }^{\circ} \mathrm{C}$ with primary antibodies, including anti-DCLK1 antibody $(1: 1,000$; Abgent AP7219b), anti-GAPDH antibody (1:2,000; Cell Signaling Technology 2118S), monoclonal mouse anti-Ecadherin antibody (1:2,000, 60335-1-Ig; Proteintech Group, Rosemont, IL, USA), monoclonal rabbit anti-N-cadherin antibody (1:2,000, D4R1H; Cell Signaling Technology), and monoclonal rabbit anti-vimentin antibody $(1: 2,000, \mathrm{D} 21 \mathrm{H} 3$; Cell Signaling Technology). Horseradish peroxidase-conjugated secondary antibodies were used to detect the primary antibodies. Protein bands were finally visualized by using FluorChem M system (ProteinSimple, San Jose, CA, USA).

\section{Overexpression and silencing of DCLKI}

The siRNA specifically targeting DCLK1 (si-DCLK1-1: 5'-CGGAGAUCGAUACUUCAAAGG-3', si-DCLK1-2: 5'-CAGAGGUGCGAGAGAAUAAGG-3') and scrambled nucleotide used as a negative control were commercially constructed (GenePharma, Shanghai, China). The plasmids were constructed as previously described ${ }^{14}$ to create transient tumor cells overexpressing DCLK1. The cells were plated and cultured until cell density reached $70 \%-80 \%$ prior to plasmids or siRNA transfection using Lipofectamine ${ }^{\mathrm{TM}} 3000$ Transfection Reagent (Thermo Fisher Scientific) following the manufacturer's protocol. The assays were conducted 48 hours posttransfection.

\section{Cell proliferation assay}

The effects of DCLK1 overexpression and inhibition on PCa cell proliferation were examined by MTT assay (KeyGEN BioTECH, Nanjing, China). The cells transfected with plasmids or siRNA were seeded in 96-well plates at a density of 3,000 cells/well. The optical density was measured by using an automatic microplate reader (Infinite M200, TECAN, Männedorf, Switzerland) at $490 \mathrm{~nm}$. 


\section{In vitro cell migration and invasion assays}

The 24-well transwell chamber (Corning Incorporated, Corning, NY, USA) coated without or with Matrige ${ }^{\circledR}$ was used to measure the ability of in vitro cell migration and invasion; $200 \mu \mathrm{L}$ cell suspension $\left(3 \times 10^{4}\right.$ cells) was loaded into the upper chamber, and $0.5 \mathrm{~mL}$ medium supplemented with 10\% FBS was added into the lower chamber. After 24 hours, the invaded cells were fixed, stained, and visualized by microscope (Olympus Optical, Tokyo, Japan). Five randomly selected fields were counted per chamber, and the results were repeated at least thrice in triplicate.

\section{Statistical analysis}

Statistical analysis was performed by using Statistical Package for the Social Science Version 20.0 (IBM Corporation, Armonk, NY, USA). Continuous parametric variables were presented as mean $\pm \mathrm{SD}$ and compared by using Student's $t$-test. The association between DCLK1 expression and clinicopathological variables was examined by Spearman correlation coefficient. The bRFS was analyzed by using the Kaplan-Meier method followed by log-rank test. Univariate and multivariate analyses using Cox proportional hazard regression model were employed to identify independent prognostic predictors of BCR. All tests of significance were two-sided, and $P<0.05$ indicated statistical significance.

\section{Results}

Increased DCLKI expression in PCa fresh samples

DCLK1 mRNA expression was assessed in 25 fresh $\mathrm{PCa}$ samples and paired BPH samples by qRT-PCR assay. On average, the relative DCLK1 mRNA level was significantly higher in tumor tissues than in BPH tissues $(P<0.001$; Figure 1A). Western blot analysis was performed simultaneously in the 25 pairs of fresh samples. Consistent with the results of qRT-PCR assay, DCLK1 protein expression was observed to be significantly elevated in PCa tissues compared with BPH $(P<0.001$; Figure $1 \mathrm{~B}$ and $\mathrm{C})$.

\section{DCLKI expression in PCa cell lines}

As shown in Figure 2, DCLK1 mRNA expression was markedly increased in PCa cell lines compared with the normal epithelial cell line RWPE-1 ( $P<0.01$; Figure 2A). Similarly, Western blot indicated that metastatic PCa cell lines (LNCaP, PC3, and DU145) had higher DCLK1 protein expression level than RWPE-1 and localized PCa cell 22Rv1, with the highest level in PC3 $(P<0.05$; Figure 2B and C).

\section{DCLKI enhances PCa cell proliferation, migration, and invasion}

To confirm the role of DCLK1 during PCa development, we altered its expression by the transfection of eukaryotic
A
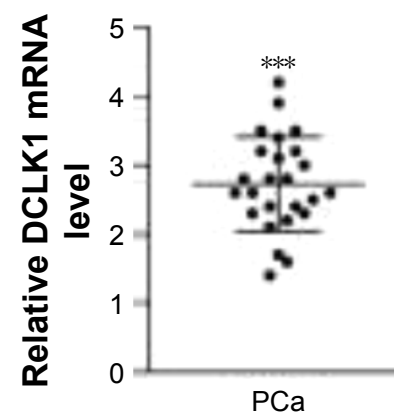

C

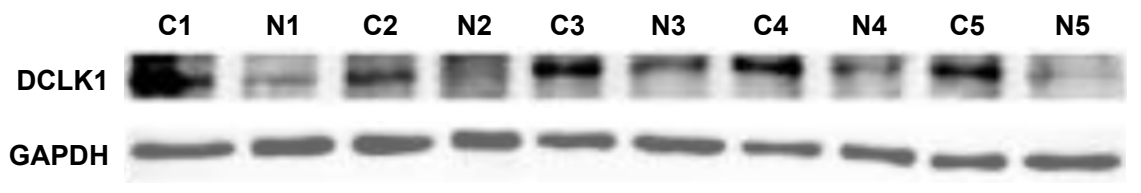

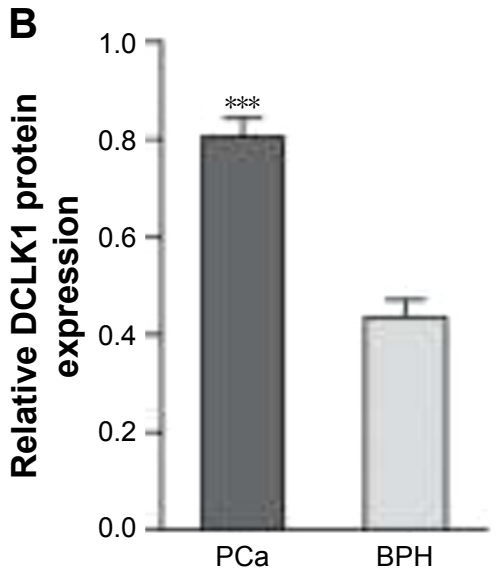

Figure I qRT-PCR and Western blot analysis of DCLKI expression in fresh PCa $(n=25)$ and BPH $(n=25)$ tissues.

Notes: (A) Relative DCLKI mRNA level was significantly higher in PCa tissues compared with BPH. Horizontal lines represent mean with SD. (B) Relative protein expression level of DCLKI/GAPDH detected by Western blot was markedly increased in PCa tissues. (C) Representative Western blot of DCLKI protein in five paired PCa $(\mathrm{C})$ and $\mathrm{BPH}(\mathrm{N})$ tissues. Data are presented as the mean $\pm \mathrm{SD}$; $* * * P<0.001$.

Abbreviations: BPH, benign prostatic hyperplasia; DCLKI, doublecortin-like kinase I; GAPDH, glyceraldehyde-3-phosphate dehydrogenase; qRT-PCR, quantitative real-time polymerase chain reaction; $\mathrm{PCa}$, prostate cancer. 


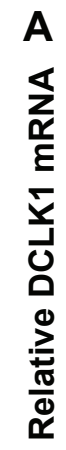
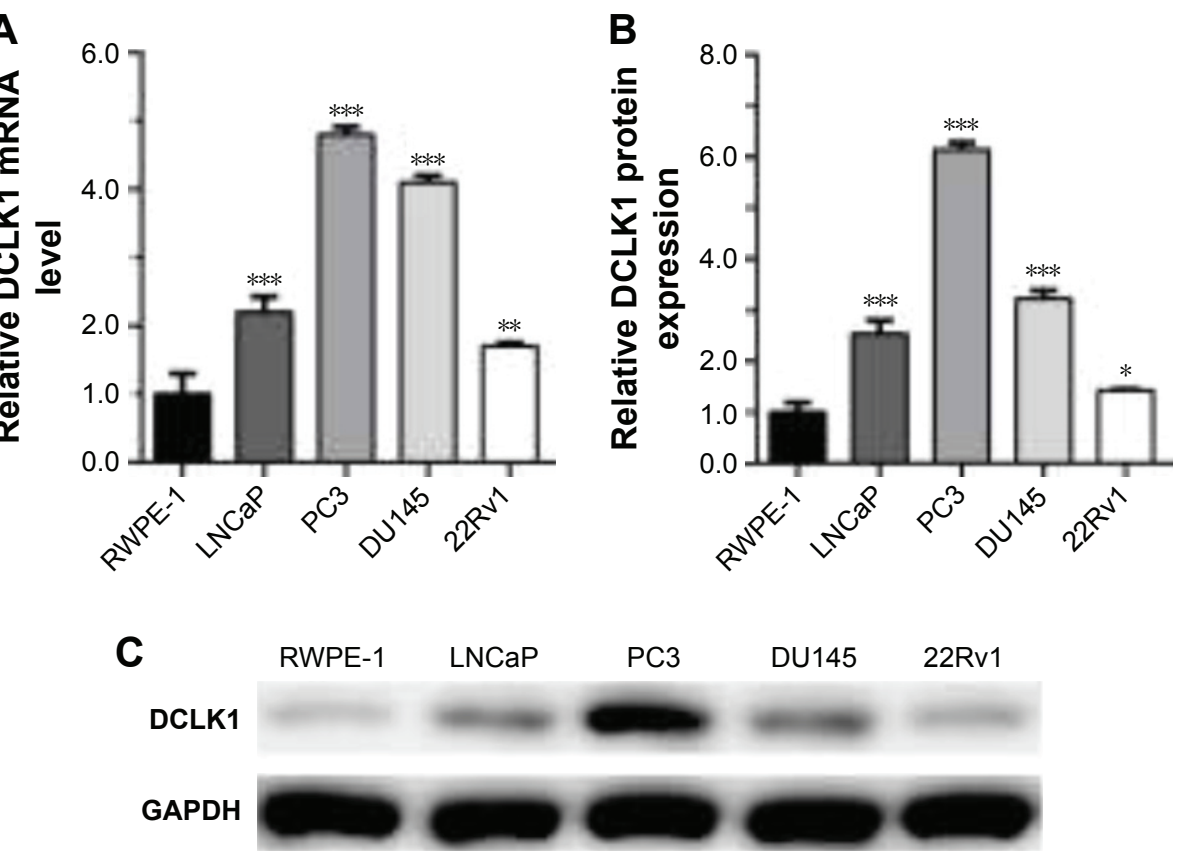

Figure 2 qRT-PCR and Western blot analysis of DCLKI expression in PCa cell lines.

Notes: (A and B) Relative DCLKI mRNA and protein expression levels were markedly increased in PCa cell lines (LNCaP, PC3, DUI 45, and 22RvI) compared with the normal epithelial cell RWPE-I. (C) Representative Western blot of DCLKI protein in cell lines. Data are presented as the mean $\pm S D$; $* P<0.05$, $* * P<0.0$ I, and $* * * P<0.001$.

Abbreviations: DCLKI, doublecortin-like kinase I; GAPDH, glyceraldehyde-3-phosphate dehydrogenase; qRT-PCR, quantitative real-time polymerase chain reaction; PCa, prostate cancer.

overexpression of plasmid and specific siRNA in two PCa cell lines 22Rv1 and PC3, respectively. Overexpression and knockdown of DCLK1 were confirmed by Western blot analysis (Figures 3A and 4A). MTT assays showed that DCLK1 overexpression promoted 22Rv1 cell proliferation, while the knockdown exhibited a contrary effect in PC3 cells $(P<0.001$; Figures 3B and 4B). In accordance with MTT assays, transwell assays demonstrated that increased DCLK1 enhanced 22Rv1 cell migration and invasion, whereas downregulation inhibited them in PC3 cells $(P<0.001$; Figures $3 \mathrm{C}$ and $4 \mathrm{C})$. Taken together, there is an obvious positive regulation of proliferation, migration,
A

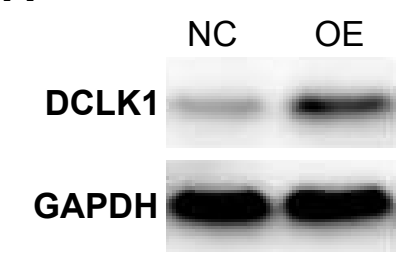

B

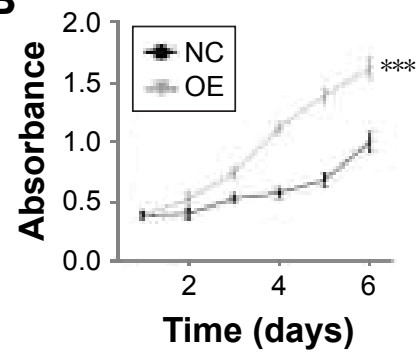

C
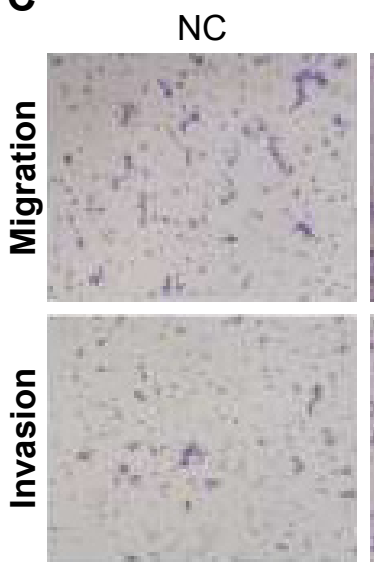

$\mathrm{OE}$
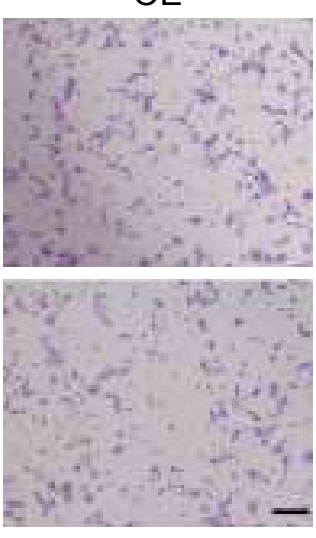

Migration

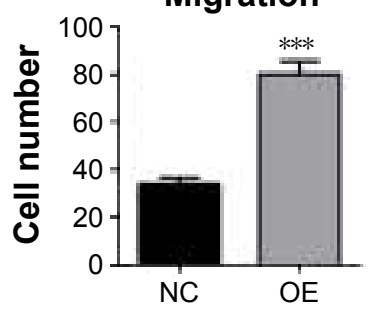

Invasion

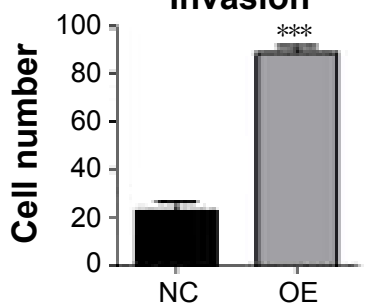

Figure 3 DCLKI overexpression enhances cell proliferation, migration, and invasion in 22Rvl cells.

Notes: (A) Western blot analysis confirmed DCLKI overexpression in $22 R v \mid$ cells. (B) MTT assays showed that DCLKI overexpression promoted 22 RvI cell proliferation. (C) Transwell assays demonstrated that increased DCLKI enhanced $22 \mathrm{RvI}$ cell migration and invasion (magnification $\times 100$ ). Data are presented as the mean \pm SD; $* * * P<0.001$.

Abbreviations: DCLKI, doublecortin-like kinase I; GAPDH, glyceraldehyde-3-phosphate dehydrogenase; NC, negative control; OE, overexpression. 
A

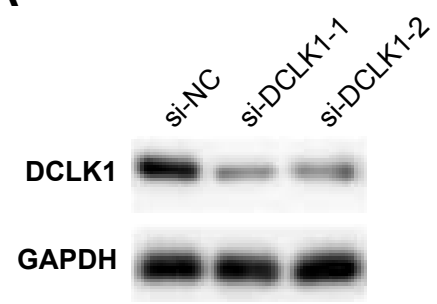

B

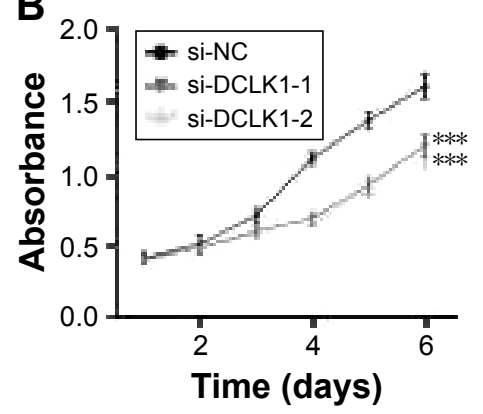

C
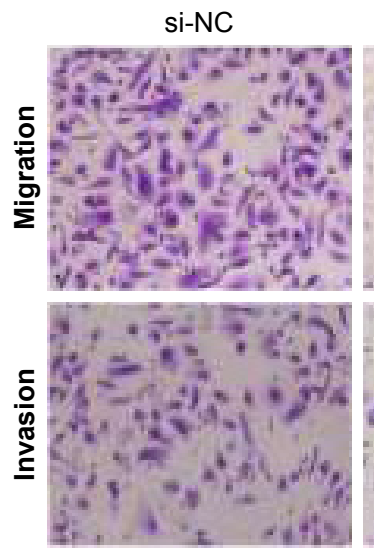

si-DCLK1-1

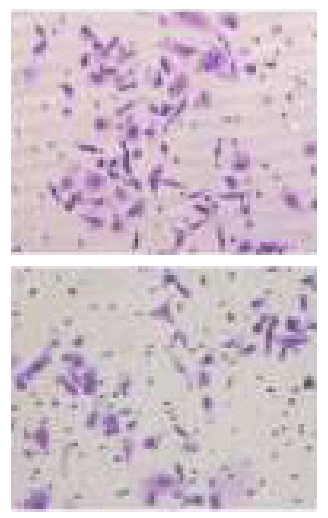

si-DCLK1-2
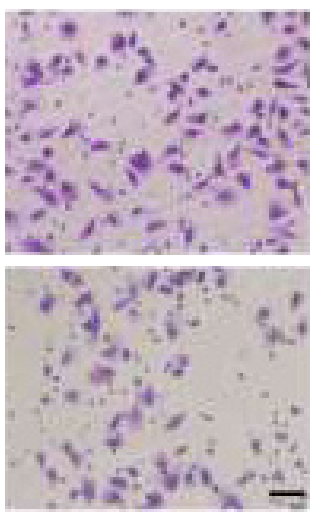

Migration
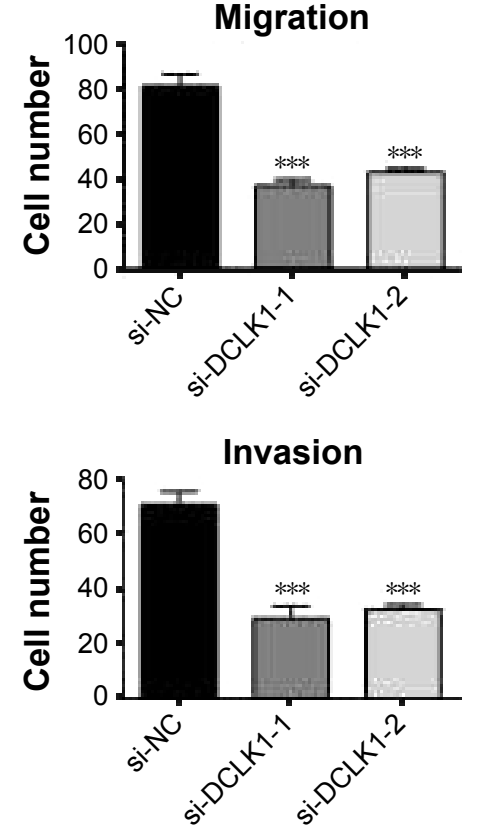

Figure 4 DCLKI downregulation inhibits cell proliferation, migration, and invasion in PC3 cells.

Notes: (A) Western blot analysis confirmed DCLKI downregulation in PC3 cells. (B) MTT assays showed that DCLKI downregulation inhibited PC3 cell proliferation. (C) Transwell assays demonstrated that decreased DCLKI inhibited PC 3 cell migration and invasion (magnification $\times 100$ ). Data are presented as the mean \pm SD; $* * * P<0.00$ I. Abbreviations: DCLKI, doublecortin-like kinase I; GAPDH, glyceraldehyde-3-phosphate dehydrogenase; NC, negative control.

and invasion by DCLK1 expression in PCa cells. Considering that DCLK1 played an important role during EMT in various other tumors, we further evaluated the effects of DCLK1 on EMT markers in PCa cell lines. The Western blot analysis demonstrated that DCLK1 knockdown increased the protein expression of E-cadherin and reduced the expression of N-cadherin and vimentin in PC 3 cells $(P<0.001$; Figure 5A and $\mathrm{C}$ ), while the overexpression exhibited a contrary effect in $22 \mathrm{Rv} 1$ cells $(P<0.001$; Figure 5A and $\mathrm{B})$. The results indicated that DCLK1 may promote tumor aggressiveness via the EMT mechanism in PCa.

\section{Overexpressed DCLKI in PCa tissues compared with $\mathrm{BPH}$ as determined by $\mathrm{IHC}$}

Positive immunoreactivity for DCLK1 was observed in the cytoplasm and nuclei of epithelial cells at various intensities and distribution levels (Figure 6A). The positive expression rates of DCLK1 in $\mathrm{PCa}$ and $\mathrm{BPH}$ tissues were $92.8 \%$ (116 of 125 ) and $52.3 \%$ (34 of $65 ; P<0.001$ ), respectively. Of the 116 PCa samples with positive DCLK1 expression, $35.2 \%$ (44 of 125) cases exhibited weak staining, $42.4 \%$ (53 of 125) moderate staining, and 15.2\% (19 of 125) strong staining (Figure 6B). In contrast, 38.5\% (25 of 65) BPH samples demonstrated weak staining, $12.3 \%$ (8 of 65) moderate staining, and $1.5 \%$ ( 1 of 65 ) strong staining (Figure $6 \mathrm{~B}$ ). The rate of patients with high DCLK1 expression (total expression score $=101-300$ ) was significantly higher in PCa than in $\mathrm{BPH}(57.6 \%$ vs $13.8 \% ; P<0.001)$.

\section{Association between DCLKI expression and clinicopathological parameters of PCa patients}

As demonstrated in Table 1, DCLK1 expression was found to be significantly correlated with postoperative Gleason grading ( $P=0.012)$, pathological T stage $(P=0.001)$, seminal vesicle 
A

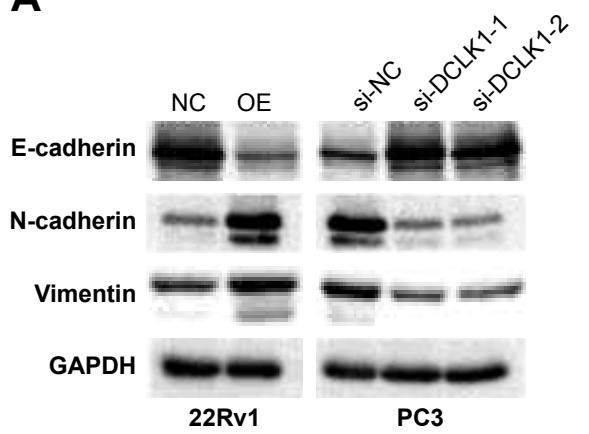

B

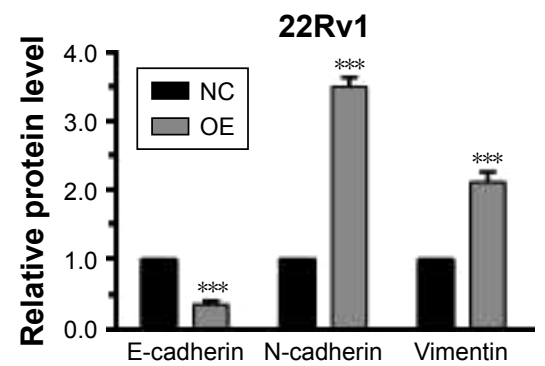

C

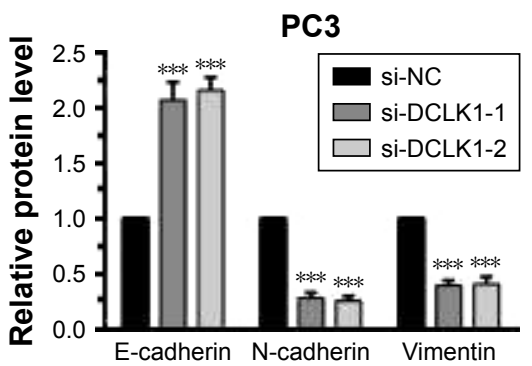

Figure 5 Western blot analysis of markers of EMT in 22RvI and PC3 cells after transfected with plasmid or siRNA.

Notes: (A) Representative Western blot bands of EMT-associated proteins. (B) DCLKI overexpression inhibited the protein expression of E-cadherin but upregulated the expression of $\mathrm{N}$-cadherin and vimentin in $22 \mathrm{Rv}$ I cells. (C) DCLKI knockdown increased the protein expression of E-cadherin while reduced the expression of $\mathrm{N}$-cadherin and vimentin in PC3 cells. Data are presented as the mean \pm SD; $* * * P<0.001$.

Abbreviations: DCLKI, doublecortin-like kinase I; EMT, epithelial-mesenchymal transition; GAPDH, glyceraldehyde-3-phosphate dehydrogenase; NC, negative control; OE, overexpression.

invasion $(P=0.026)$, and lymph node involvement $(P=0.017)$. However, no significant correlation was observed between DCLK1 expression and age $(P=0.799)$, preoperative PSA $(P=0.066)$, as well as surgical margin status $(P=0.909)$.

\section{Predictive value of DCLKI expression on BCR}

The median follow-up was 6.4 years with a maximum follow-up of 9.6 years. During the follow-up period, $41.7 \%$ (30 of 72) PCa patients with high DCLK1 expression demonstrated BCR, while the BCR rate in patients with low DCLK1 expression was $13.2 \%$ ( 7 of 53; $P=0.001$ ). As demonstrated in Table 2, both univariate and multivariate Cox proportional hazard analyses indicated that postoperative Gleason grading, pathological T stage, seminal vesicle invasion, lymph node involvement, and DCLK1 expression were independent predictors of BCR after RP. Since DCLK1 expression was strongly associated with postoperative Gleason grading, pathological T stage, seminal vesicle invasion, and lymph node involvement, data were further examined by stratified models, aiming at confirming predictive value independent of the parameters mentioned above. We also performed the Cox regression analysis according to the subgroups of the associated parameters (data not shown). The $P$-values for DCLK1 expression in BCR prediction in these stratified factors were 0.038 (postoperative Gleason grading), 0.013 (pathological T stage), 0.013 (seminal vesicle invasion), and 0.016 (lymph node involvement), respectively, which suggested that DCLK1 was an independent predictor of BCR. As shown in Figure 6C, patients with high DCLK1 expression had remarkably lower bRFS than those with low DCLK1 expression. The 5-year bRFS rates for patients with high DCLK1 expression and those with low DCLK1 expression were $58.9 \%$ and $88.4 \%$, respectively $(P<0.001)$.
A

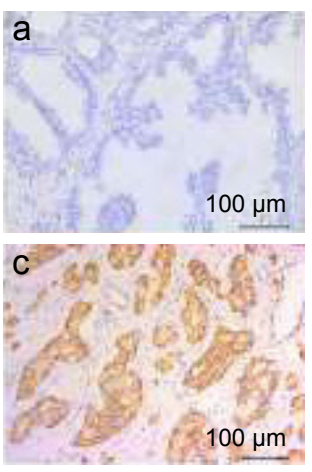

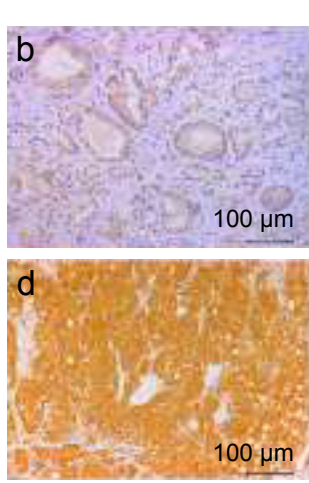

B

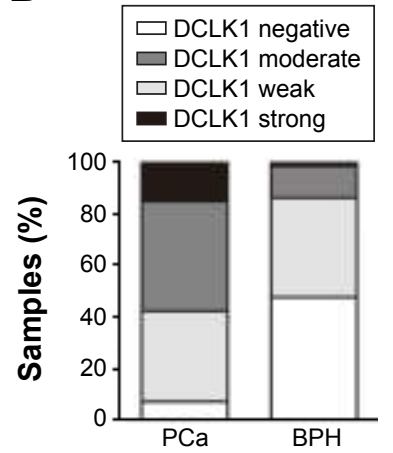

C

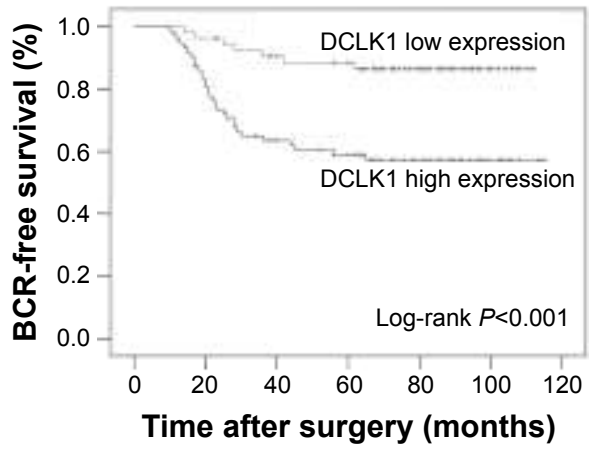

Figure 6 DCLKI expression determined by IHC and its correlation with BCR-free survival.

Notes: (A) Representative images of DCLKI staining in BPH and PCa: (a) BPH tissue showed no DCLKI staining; (b-d) PCa tissues showed faint/equivocal or focal (b), intermediate (c), and bright (d) DCLKI staining, respectively (magnification $\times 200)$. (B) Distribution of DCLKI total expression score for PCa $(n=125)$ and BPH ( $=65)$ tissues. (C) Kaplan-Meier survival curves of BCR-free survival based on DCLKI expression in patients with PCa.

Abbreviations: BCR, biochemical recurrence; BPH, benign prostatic hyperplasia; DCLKI, doublecortin-like kinase I; IHC, immunohistochemistry; PCa, prostate cancer. 
Table 2 Univariate and multivariate analyses of prognostic factors for biochemical recurrence

\begin{tabular}{|c|c|c|c|c|}
\hline \multirow[t]{2}{*}{ Variables } & \multicolumn{2}{|l|}{ Univariate } & \multicolumn{2}{|l|}{ Multivariate } \\
\hline & HR (95\% Cl) & $P$-value & HR ( $95 \% \mathrm{Cl})$ & $P$-value \\
\hline Age (years) & & 0.550 & - & - \\
\hline$<68$ & I (reference) & & & \\
\hline$\geq 68$ & $1.22(0.64-2.33)$ & & & \\
\hline Preoperative PSA (ng/mL) & & 0.689 & - & - \\
\hline$<10$ & I (reference) & & & \\
\hline $10-20$ & $1.29(0.46-3.58)$ & 0.626 & & \\
\hline$>20$ & $\mathrm{I} .52(0.57-4.1 \mathrm{I})$ & 0.404 & & \\
\hline Postoperative Gleason grading & & 0.039 & & 0.018 \\
\hline Grade group I & I (reference) & & I (reference) & \\
\hline Grade groups 2 and 3 & $1.82(0.79-4.22)$ & 0.161 & $2.67(1.11-6.45)$ & 0.029 \\
\hline Grade groups 4 and 5 & $2.83(1.27-6.30)$ & 0.011 & $3.19(1.39-7.34)$ & 0.006 \\
\hline Pathological T stage & & $<0.001$ & & $<0.00 \mathrm{I}$ \\
\hline $\mathrm{T} 2$ & I (reference) & & I (reference) & \\
\hline T3a & $4.86(2.14-11.03)$ & $<0.00$ I & $3.87(1.60-9.32)$ & 0.003 \\
\hline T3b & $17.90(7.22-44.38)$ & $<0.00$ I & $19.96(6.91-57.65)$ & $<0.00$ I \\
\hline Seminal vesicle invasion & & $<0.001$ & & 0.012 \\
\hline No & I (reference) & & I (reference) & \\
\hline Yes & $4.68(2.34-9.39)$ & & $2.54(1.23-5.28)$ & \\
\hline Lymph node involvement & & $<0.001$ & & 0.014 \\
\hline No & I (reference) & & I (reference) & \\
\hline Yes & $3.86(1.98-7.55)$ & & $2.40(1.20-4.83)$ & \\
\hline Positive surgical margin & & 0.849 & - & - \\
\hline No & I (reference) & & & \\
\hline Yes & $0.92(0.38-2.20)$ & & & \\
\hline DCLKI expression & & 0.001 & & 0.014 \\
\hline Low & I (reference) & & I (reference) & \\
\hline High & $4.00(1.75-9.12)$ & & $2.98(1.25-7.14)$ & \\
\hline
\end{tabular}

Abbreviations: DCLKI, doublecortin-like kinase I; HR, hazard ratio; PSA, prostate-specific antigen.

\section{Discussion}

Although PSA remains the most generally used biomarker for PCa screening and diagnosis, its sensitivity and specificity are limited, which may lead to false-positive diagnoses and excessive treatment. ${ }^{15}$ Localized PCa patients with similar clinicopathological parameters and therapeutic regimen may yet present different clinical outcomes, such as BCR and tumor metastasis. In other words, as the heterogeneous genetic background of PCa, its biological and clinical behavior cannot be reliably predicted by conventional diagnostic or prognostic markers. ${ }^{16}$ Therefore, more efforts are required to seek novel and effective biomarkers of initiation, progression, and prognosis in $\mathrm{PCa}$.

To date, the expression of DCLK1 in PCa has been previously mentioned in only two publications. ${ }^{13,17}$ The IHC analyses on human multicancer tissue microarrays performed by Sureban et $\mathrm{al}^{17}$ showed that minimal or no DCLK1 immunoreactivity was detected in normal prostate, while overexpressed DCLK1 was found in the cytoplasm of PCa epithelial cells. In addition, Roudier et a ${ }^{13}$ revealed that DCLK1 was upregulated at the protein level in unpaired
$\mathrm{ERG}+$ primary PCa and metastatic castration-resistant PCa. However, the association between DCLK1 expression and the clinicopathological features of PCa patients, as well as the prognostic implication of DCLK1 in PCa, has not yet been clarified.

In the present study, we found that DCLK1 expression was markedly elevated in PCa tissues compared with BPH. Similarly, DCLK1 expression was remarkably increased in PCa cell lines than in normal prostatic epithelial cell. Although PC3 and DU145 demonstrated similar DCLK1 mRNA level, their protein expression showed a divergence, which may resulted from different processes of posttranslational modification. In PCa cell lines, DCLK1 enhanced cell proliferation, migration, and invasion, and its expressions in metastatic cells were significantly increased compared with localized cells, suggesting that DCLK1 may promote PCa metastasis. We also found that upregulated DCLK1 was associated with advanced clinicopathological features in patients with PCa. Furthermore, we identified DCLK1 as an independent predictor of BCR after RP. These results suggest that the upregulation of DCLK1 indicates biologically more 
aggressiveness and may be an underlying initiating factor of PCa development. Accordingly, the expression level of DCLK1 may contribute to differentiating PCa from $\mathrm{BPH}$ and accurately identifying patients who would benefit from early intervention.

The molecular mechanisms that account for the association between DCLK1 overexpression and PCa aggressiveness and BCR are unclear. However, as a marker of intestinal and pancreatic CSCs, DCLK1 has been proposed to be critical for cancer initiation, growth, EMT, and metastasis of various other cancers. ${ }^{5-8}$ Strikingly, the critical signaling pathways NF- $\mathrm{KB}, \mathrm{WNT}$, PTEN/PI3K/AKT, and $\mathrm{NOTCH}$ that are involved in both CSCs and EMT have been revealed to be regulated by DCLK1 expression in cancer models. ${ }^{5,17-19}$ Previously, the presence of CSCs has also been demonstrated in $\mathrm{PCa}$, which can initiate tumor by differentiating into heterogeneous lineages of cancer cells that comprise the whole tumor. ${ }^{20}$ Furthermore, direct inhibition of WNT, NOTCH, PTEN/PI3K/AKT, and other cell signaling pathways has resulted in tumor suppressive effect via lowering the population of prostate CSCs. ${ }^{21,22}$ To be note, BCR represents an elevated PSA value, and the expression of PSA is principally activated by androgen and regulated by the androgen receptor (AR) signaling at the transcriptional level. Accumulating researches have indicated that AR is a downstream regulatory factor of the critical cell signaling pathways mentioned above. For instance, one coactivator that remarkably influences the transcriptional activity of human $\mathrm{AR}$ is $\beta$-catenin, which is a pivotal molecule in the canonical WNT signaling pathway. ${ }^{23}$ In addition, Yang et $\mathrm{al}^{24}$ found that the WNT signaling pathway could modulate the levels of human AR-encoded protein through an ubiquitin-mediated degradation mechanism controlled by $\mathrm{AKT} /$ protein kinase B signaling. Moreover, the PI3K-AKT-mTOR pathway is thought to be dominant over AR signaling in PCa cells, ${ }^{25}$ and both AKT activation and PTEN loss are associated with BCR following RP. ${ }^{26,27}$ In the present study, we have also proved that DCLK1 could regulate the markers of EMT in PCa cells. Taken together, we speculated that DCLK1 may promote tumor initiation, growth, progression, and metastasis of PCa by the enhancement of CSCs and EMT properties, and upregulated DCLK1 might promote BCR via AR signaling activated by WNT or PTEN/PI3K/AKT signaling pathway. However, further investigations are still required to verify the abovementioned underlying mechanisms.

There are certain limitations in our study. First, it was just conducted as a retrospective analysis of the PCa cases from one single institution. Second, the sample volume of
125 PCa patients is relatively small as we strictly selected the participants to control the potential heterogeneity. However, it is enough to guarantee statistical significance. Third, research on the role of DCLK1-induced oncogenesis in PCa was absent in this study. Therefore, further studies are needed to elucidate the molecular mechanisms of DCLK1 expression in the tumorigenesis and progression of $\mathrm{PCa}$.

\section{Conclusion}

In summary, we, for the first time, explored the expression pattern of DCLK1 in PCa and its correlation with clinicopathological features, as well as its prognostication of BCR after RP. Our data suggested that overexpressed DCLK1 in $\mathrm{PCa}$ tissues was significantly correlated with unfavorable clinicopathological characteristics and bRFS of patients. DCLK1 might serve as a potential biomarker for risk stratification and prognosis of patients with $\mathrm{PCa}$, and it may help clinicians to render more rational and efficient therapeutic strategies for PCa patients.

\section{Acknowledgments}

This study was supported by grants from National Natural Science Foundation of China (81772754 and 81372731), the Science and Technology Program of Guangzhou, China (201607010187), the Fundamental Research Funds for the Central Universities (16ykjc20), and the key research project of Natural Science Foundation of Guangdong, China (2017A03038009).

\section{Disclosure}

The authors report no conflicts of interest in this work.

\section{References}

1. Chen W, Zheng R, Baade PD, et al. Cancer statistics in China, 2015. CA Cancer J Clin. 2016;66(2):115-132.

2. Boorjian SA, Eastham JA, Graefen M, et al. A critical analysis of the long-term impact of radical prostatectomy on cancer control and function outcomes. Eur Urol. 2012;61(4):664-675.

3. Filella X, Foj L. Prostate cancer detection and prognosis: from prostate specific antigen (PSA) to exosomal biomarkers. Int J Mol Sci. 2016; 17(11):pii:E1784.

4. Shin E, Kashiwagi Y, Kuriu T, et al. Doublecortin-like kinase enhances dendritic remodelling and negatively regulates synapse maturation. Nat Commun. 2013;4:1440.

5. Chandrakesan P, Weygant N, May R, et al. DCLK1 facilitates intestinal tumor growth via enhancing pluripotency and epithelial mesenchymal transition. Oncotarget. 2014;5(19):9269-9280.

6. Weygant N, Qu D, Berry WL, et al. Small molecule kinase inhibitor LRRK2-IN-1 demonstrates potent activity against colorectal and pancreatic cancer through inhibition of doublecortin-like kinase 1. Mol Cancer. 2014;13:103

7. Ali N, Chandrakesan P, Nguyen CB, et al. Inflammatory and oncogenic roles of a tumor stem cell marker doublecortin-like kinase (DCLK1) in virus-induced chronic liver diseases. Oncotarget. 2015;6(24): 20327-20344. 
8. Weygant N, Qu D, May R, et al. DCLK1 is a broadly dysregulated target against epithelial-mesenchymal transition, focal adhesion, and stemness in clear cell renal carcinoma. Oncotarget. 2015;6(4): 2193-2205.

9. Vega KJ, May R, Sureban SM, et al. Identification of the putative intestinal stem cell marker doublecortin and CaM kinase-like-1 in Barrett's esophagus and esophageal adenocarcinoma. J Gastroenterol Hepatol. 2012;27(4):773-780.

10. Buyyounouski MK, Choyke PL, McKenney JK, et al. Prostate cancer - major changes in the American Joint Committee on Cancer eighth edition cancer staging manual. CA Cancer J Clin. 2017;67(3): 245-253.

11. Epstein JI, Egevad L, Amin MB, et al. The 2014 International Society of Urological Pathology (ISUP) Consensus Conference on Gleason Grading of Prostatic Carcinoma: definition of grading patterns and proposal for a new grading system. Am J Surg Pathol. 2016;40(2): 244-252.

12. Cornford P, Bellmunt J, Bolla M, et al. EAU-ESTRO-SIOG Guidelines on Prostate Cancer. Part II: treatment of relapsing, metastatic, and castration-resistant prostate cancer. Eur Urol. 2017;71(4): 630-642.

13. Roudier MP, Winters BR, Coleman I, et al. Characterizing the molecular features of ERG-positive tumors in primary and castration resistant prostate cancer. Prostate. 2016;76(9):810-822.

14. Li K, Pang J, Cheng H, et al. Manipulation of prostate cancer metastasis by locus-specific modification of the CRMP4 promoter region using chimeric TALE DNA methyltransferase and demethylase. Oncotarget. 2015;6(12):10030-10044.

15. Carter HB, Albertsen PC, Barry MJ, et al. Early detection of prostate cancer: AUA Guideline. J Urol. 2013;190(2):419-426.

16. Wei L, Wang J, Lampert E, et al. Intratumoral and intertumoral genomic heterogeneity of multifocal localized prostate cancer impacts molecular classifications and genomic prognosticators. Eur Urol. 2017; 71(2):183-192.
17. Sureban SM, May R, Mondalek FG, et al. Nanoparticle-based delivery of siDCAMKL-1 increases microRNA-144 and inhibits colorectal cancer tumor growth via a Notch-1 dependent mechanism. J Nanobiotechnology. 2011;9:40.

18. Sureban SM, May R, Qu D, et al. DCLK1 regulates pluripotency and angiogenic factors via microRNA-dependent mechanisms in pancreatic cancer. PLoS One. 2013;8(9):e73940.

19. Chandrakesan P, Panneerselvam J, Qu D, et al. Regulatory roles of Dclk1 in epithelial mesenchymal transition and cancer stem cells. J Carcinog Mutagen. 2016;7(2):pii:257.

20. Harris KS, Kerr BA. Prostate cancer stem cell markers drive progression, therapeutic resistance, and bone metastasis. Stem Cells Int. 2017; 2017:8629234.

21. Dubrovska A, Elliott J, Salamone RJ, et al. Combination therapy targeting both tumor-initiating and differentiated cell populations in prostate carcinoma. Clin Cancer Res. 2010;16(23):5692-5702.

22. Rybak AP, Bristow RG, Kapoor A. Prostate cancer stem cells: deciphering the origins and pathways involved in prostate tumorigenesis and aggression. Oncotarget. 2015;6(4):1900-1919.

23. Yang F, Li X, Sharma M, et al. Linking beta-catenin to androgensignaling pathway. J Biol Chem. 2002;277(13):11336-11344.

24. Yang X, Chen MW, Terry S, et al. Complex regulation of human androgen receptor expression by Wnt signaling in prostate cancer cells. Oncogene. 2006;25(24):3436-3444.

25. Kaarbo M, Mikkelsen OL, Malerod L, et al. PI3K-AKT-mTOR pathway is dominant over androgen receptor signaling in prostate cancer cells. Cell Oncol. 2010;32(1-2):11-27.

26. Bedolla R, Prihoda TJ, Kreisberg JI, et al. Determining risk of biochemical recurrence in prostate cancer by immunohistochemical detection of PTEN expression and Akt activation. Clin Cancer Res. 2007;13(13):3860-3867.

27. Ayala G, Thompson T, Yang G, et al. High levels of phosphorylated form of Akt-1 in prostate cancer and non-neoplastic prostate tissues are strong predictors of biochemical recurrence. Clin Cancer Res. 2004; 10(19):6572-6578
OncoTargets and Therapy

\section{Publish your work in this journal}

OncoTargets and Therapy is an international, peer-reviewed, open access journal focusing on the pathological basis of all cancers, potential targets for therapy and treatment protocols employed to improve the management of cancer patients. The journal also focuses on the impact of management programs and new therapeutic agents and protocols on

\section{Dovepress}

patient perspectives such as quality of life, adherence and satisfaction The manuscript management system is completely online and includes a very quick and fair peer-review system, which is all easy to use. Visit http://www.dovepress.com/testimonials.php to read real quotes from published authors. 